\title{
Discussion of 010405JOR by J. Kim et al.
}

Citation: Journal of Rheology 58, 1391 (2014); doi: 10.1122/1.4892817

View online: https://doi.org/10.1122/1.4892817

View Table of Contents: http://sor.scitation.org/toc/jor/58/5

Published by the The Society of Rheology

\section{Articles you may be interested in}

Discussion of $004405 J O R$ by M. Laurati et al.

Journal of Rheology 58, 1418 (2014); 10.1122/1.4892815

Thermokinematic memory and the thixotropic elasto-viscoplasticity of waxy crude oils Journal of Rheology 61, 427 (2017); 10.1122/1.4978259

A micro-mechanical study of coarsening and rheology of colloidal gels: Cage building, cage hopping, and Smoluchowski's ratchet

Journal of Rheology 58, 1121 (2014); 10.1122/1.4892115

Discussion of 019405JOR by R. Zia et al.

Journal of Rheology 58, 1158 (2014); 10.1122/1.4892821

Linking self-assembly, rheology, and gel transition in attractive colloids

Journal of Rheology 58, 1219 (2014); 10.1122/1.4878838

Direct observation of DNA dynamics in semidilute solutions in extensional flow Journal of Rheology 61, 151 (2017); 10.1122/1.4972236

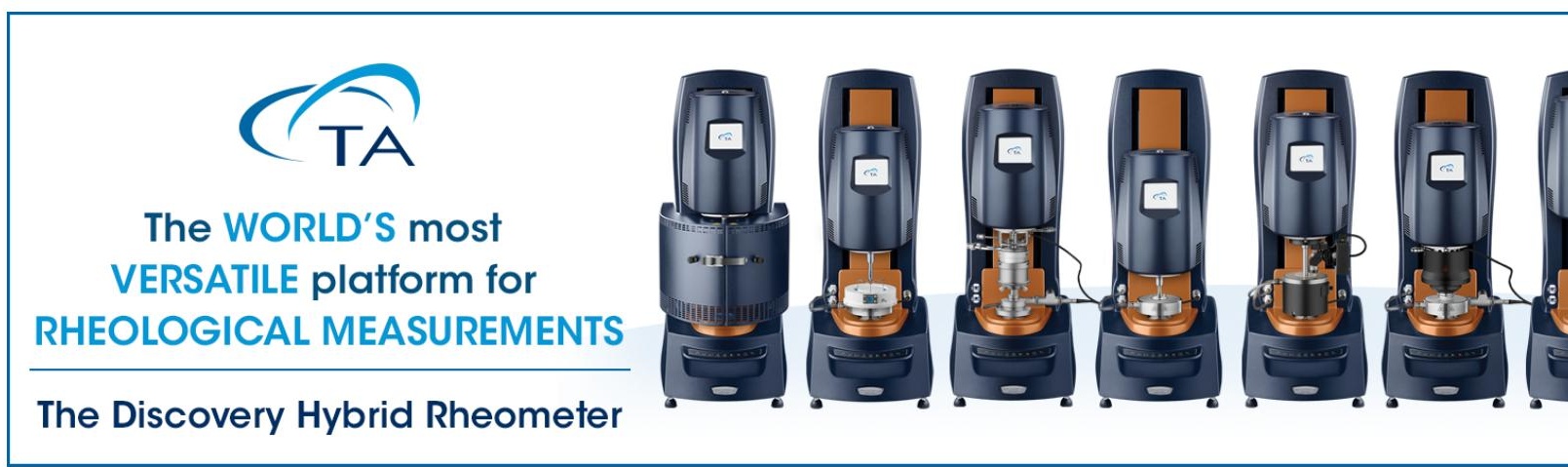




\section{Discussion of 010405JOR by J. Kim et al.}

Question: Alessio Zaccone: How does the proposed structural breakdown mechanism differentiate itself from previously proposed mechanisms based on shear-induced severing of weak connections between clusters?

Author Response: As discussed in Sec. IV, we propose that the dominant microstructural processes accompanying yielding are:

1. In the initial stages of yielding, compression of the internal structure (and corresponding formation of large voids) of the particle-rich domains, which appears to dominate the nonlinear mechanical response in regions I and II. Such a process does not require net breakage of intercluster bonds, but merely rearrangements of same in order to form a more compact structure. This could be seen as akin to the initial stage of the "two-step" mechanisms previously proposed, such as by Koumakis and Petekidis (2011), in which local bond rearrangement occurs within clusters. However, our measurements establish that these rearrangements primarily occur at length scales much larger than individual particles, and that the internal fractal microstructure of clusters remains relatively unchanged (as evident in the SANS data for $q>0.1 \AA^{-1}$ ) for the case of a heterogeneous gel.

2. In the final stage of yielding (which dominates in region III), the network compression saturates and both decorrelation (indicated by a disappearance of the low- $q$ peak), and "depercolation" (indicated by a maximum in the homogeneity of cluster-cluster correlations) of cluster-cluster interactions occurs. We think this process is indeed the same as that put forth by others-including the work by Hsiao et al. (2012)—in which breakage of weak intercluster bonds allows clusters to be suspended and flow, but with an associated length scale coinciding with the phase separated structure rather than the local particle-particle scale.

To summarize, the microstructural processes we propose here to accompany yielding are qualitatively similar to those put forth previously by others. However, our measurements clearly show that, at least for the heterogeneous gels studied here, these processes are primarily occurring at much larger length scales than the local scale of particleparticle bonds that is usually depicted in these hypothesized mechanisms. This is perhaps a subtle difference, but one that we believe has significant implications for the rheological modeling of these systems near the yield point.

Question: Marco Laurati: In manuscript 004405JOR of this same special issue, we show that concentrated depletion gels formed by hard-sphere like colloidal particles do not show the pronounced in-cycle yielding observed for these samples, i.e., the stress overshoot close to strain reversal in the Lissajous figures of Fig. 9. Still we speculate that microstructural processes relatively similar to the ones proposed here may characterize yielding in our depletion gels. Could then the stronger in-cycle yielding observed for this system be in part associated with the larger softness of the nanoemulsion investigated here? This effect of the particle softness has been recently pointed out in a manuscript by Koumakis et al. (2012). 
Author Response: This is certainly a possibility, although we note that even the "hard" PMMA particle system studied in the work by Koumakis and coworkers also exhibits a significant stress overshoot (but it is exaggerated for softer particles). For reference, one might wish to compare the relative "softness" of the particles between our work and that of Koumakis and coworkers. The effective elastic modulus of our droplets (estimated to be equal to the Laplace pressure, $\gamma / a$ ) is approximately $0.5 \mathrm{MPa}$. This is obviously much softer than PMMA ( 2-3 GPa) but is still two orders of magnitude stiffer than the elastic modulus of PNIPAM microgel particles (5-10 kPa) measured by Hashmi and Dufresne (2009).

It is thus unclear a priori how much deformation of the nanodroplets one would expect during yielding. To estimate the potential amount of deformation, we calculate the capillary number, characterizing the magnitude of viscoelastic stress relative to the Laplace pressure, at the local stress maximum, $C a=\sigma_{c} a / \gamma$, where $\gamma$ is the interfacial tension of the two fluids. In our measurements, $C a$ is vanishingly small, $O\left(10^{-7}\right)$, due to the nanoscopic size of the droplets. In this limit, simple theories for droplet deformation in viscous flows [Maffetone and Minale (1998)] would predict for this capillary number and the viscosities of the two liquids a relative deformation of the droplets less than $0.0001 \%$, much smaller than the accumulated strain on the material at the yield point. Indeed, we see no observable anisotropy in the 2D rheo-SANS patterns at high $q$-values that would indicate droplet deformation, even at the highest strain amplitudes studied. We therefore conclude that there is no significant deformation of the droplets under conditions relevant to the initial yielding of these gels.

Question: Ralph Colby: Since Fig. 1a shows an acute change in response when changing temperature by $1 \mathrm{~K}$, can you tell us how much does the temperature of the sample rise during LAOS?

Author Response: For the LAOS experiments, the temperature was maintained at $50.0 \pm 0.15^{\circ} \mathrm{C}$ using a circulating bath connected to the lower plate. The temperature stability during measurement shows a small initial increase of small initial increase of $\sim 0.05^{\circ} \mathrm{C}$ during a typical dynamic strain sweep due to the small remaining transient during heating. We first note that this temperature is relatively deep in the gel region ( $T_{\text {gel }} \sim 44{ }^{\circ} \mathrm{C}$ for this sample), where the linear moduli are not so acutely dependent on temperature. Using these same conditions, we found that the nonlinear viscoelasticity of the sample was highly reproducible despite any temperature fluctuations. As such, we do not believe small temperature fluctuations due to the precision of the temperature control will produce significant changes in the measured rheology for these conditions.

Nevertheless, it is possible that the sample experiences temperature excursions due to viscous heating not measured by the rheometer, since the temperature is measured inside the lower plate. To estimate the magnitude of these effects, we calculate the Brinkman number, which for oscillatory shear in a cone-plate geometry can be defined as

$$
\mathrm{Br}=\frac{\eta\left(\dot{\gamma}_{0}\right) \mathrm{H}^{2} \dot{\gamma}_{0}^{2}}{\mathrm{k}\left(\mathrm{T}_{\mathrm{w}}-\mathrm{T}_{\mathrm{f}}\right)},
$$

where $\dot{\gamma}_{0}$ is the shear rate amplitude $\left(\omega \gamma_{0}\right), H$ is the gap height at the cone edge, $k$ is the thermal conductivity of the fluid (assumed here to be approximately that of water), and $T_{w}$ and $T_{f}$ are the temperatures of the wall and bulk fluid, respectively. As a worst case, we assume the thermal driving force for heat transfer, $T_{w}-T_{f}$, to be on the order of the 
precision of the temperature control, $0.15^{\circ} \mathrm{C}$. For these conditions and the ranges of shear rate amplitude and viscosity we measure, we calculate $B r \sim O\left(10^{-4}-10^{-2}\right)$. Therefore, we assume any temperature excursions due to viscous heating are negligible.

Question: Ralph Colby: You correctly point out that the slope of -1 in Fig. 8(c) indicates a shear stress independent of shear rate (a yield stress) but what does the steeper slope at lower shear rates in Fig. 8(c) mean?

Author Response: To remind the reader, the instantaneous viscosity plotted in Fig. 8 (c) is not the steady shear viscosity, but rather an apparent viscosity, $\eta_{L}$, defined at the point of maximum instantaneous shear rate, $\omega \gamma(t)$, in the Lissajous curve. The steeper slope at strain rate amplitudes near the onset of region II is due to the influence of the location of the stress overshoot relative to the point of maximum instantaneous shear rate (i.e., zero instantaneous strain) at which the instantaneous viscosity is defined. As shown in Fig. 5(c) for such strain amplitudes (dark blue curve), the instantaneous stress is actually decreasing at the point of maximum instantaneous shear rate. This is what causes the slope in Fig. 8(c) to be greater than -1 for this portion of region II. As such, the particular definition of $\eta_{L}$ we choose is ill-posed in this regime, as the gel is not yet in the fully flowing portion of the intracycle response. Therefore, $\eta_{L}$ values for strain amplitudes near the onset of region II should not be interpreted as "true" viscosities in the strict sense, but rather simply a relative measure of the viscous response in the process immediately preceding flow.

A better definition of $\eta_{L}$ would perhaps be at the instantaneous shear rate corresponding to the local stress minimum, which is not observed at strain amplitudes close to the onset of region II. We note that these two definitions produce approximately identical results for strain amplitudes deeper into region II, since the local stress minimum (once established) falls nearly at the point of maximum shear rate. However, since the present definition has some precedent [Ewoldt et al. (2008)], we use it so that it is comparable with previously reported literature.

Dimitris Vlassopoulos: The yielding studies in colloidal glasses and gels clearly show the gradual nature of the yielding process, as stated by the authors also supported by recent simulation studies. In this respect, two-step yielding can be viewed as a generic term pointing to more than one relevant length scales in the system. The present result appears to fit in this framework and so one wonders to what degree the "three-stage" is system specific or not. In other words, the gel yields gradually (starting from the larger length scales) as shown in colloid-polymer systems.

Author Response: Indeed, our results are consistent with the framework that multistage yielding arises in materials with hierarchical structures and dynamic processes. In particular, we believe our results should be general to gels and glasses with significant arrested structural heterogeneity, which in this case is homogenized before the eventual transition to flow. However, there are still some features of the mechanical response, such as the local maximum during intracycle yielding as well as the frequencydependence of various nonlinear measures, which may depend on the particular features of the system.

We also emphasize that the complex rheological signatures accompanying these hierarchical processes under LAOS deformation are best elucidated through intracycle analysis of the full nonlinear response, which shows distinct transitions between different stages of yielding, as opposed to intercycle analysis of linearized 
moduli, in which these transitions appear more gradual due to averaging of the intracycle response.

Dimitris Vlassopoulos: In addition, one wonders whether the "sequence of physical processes" methodology (which was proposed in the context of dense nearly nonthixotropic systems) is valid for systems such as the present gel, which is thixotropic and hence often the structural time scale exceeds the period of a cycle. In that case, the concept of the above sequence is violated.

Author Response: There is certainly thixotropy in the system. However, as we discuss in Sec. III C, the thixotropy observed in the Lissajous curves occurs over time scales far longer than a single cycle (as is evident in Fig. 4). Therefore, our LAOS measurements can be approximated as probing a quasistatic state of the system at the beginning of the thixotropic response. As such, we believe that the SPP approach (i.e., the calculation and interpretation of the instantaneous moduli $R^{\prime}$ and $R^{\prime \prime}$ and nonlinear measures calculated there from) is valid in the sense that the processes so identified are fast processes that relax within one cycle and are much faster than the underlying thixotropy.

We do caution, however, that the analogy between the intracycle response in LAOS and steady shear responses that held for other systems, such as reported by Rogers et al. (2011b), is certainly violated in the present system for exactly the reasons you point out. A more comprehensive characterization of the thixotropic would thus require detailed measurements under startup of steady shear, which we leave for future studies.

\section{References}

Ewoldt, R. H., A. E. Hosoi, and G. H. McKinley, "New measures for characterizing nonlinear viscoelasticity in large amplitude oscillatory shear," J. Rheol. 52, 1427-1458 (2008).

Hashmi, S. M., and E. R. Dufresne, "Mechanical properties of individual microgel particles through the deswelling transition," Soft Matter 5, 3682-3688 (2009).

Hsiao, L. C., R. S. Newman, S. C. Glotzer, and M. J. Solomon, "Role of isostaticity and load-bearing microstructure in the elasticity of yielded colloidal gels," PNAS 109(40), 16029-16034 (2012).

Koumakis, N., and G. Petekidis, "Two step yielding in attractive colloids: transition from gels to attractive glasses," Soft Matter 7(6), 2456-2470 (2011).

Koumakis, N., A. Pamvouxoglou, A. S. Poulos, and G. Petekidis, "Direct comparison of the rheology of model hard and soft particle glasses," Soft Matter 8, 4271-4284 (2012).

Maffetone, P. L., and M. Minale, "Equation of change for ellipsoidal drops in viscous flow," J. Non-Newtonian Fluid Mech. 78, 227-241 (1998).

Rogers, S. A., B. M. Erwin, D. Vlassopoulos, and M. Cloitre, "A sequence of physical processes determined and quantified in LAOS: Application to a yield stress fluid," J. Rheol. 55, 435-458 (2011). 